Proceedings

\title{
Cyclodextrin-Efavirenz Complexes Investigated by Solid State and Solubility Studies ${ }^{+}$
}

\author{
Susana Santos Braga ${ }^{1, *}$, Karyna Lysenko ${ }^{1}$, Firas El-Saleh ${ }^{2}$ and Filipe A. Almeida Paz ${ }^{3}$ \\ 1 LAQV-REQUIMTE and Department of Chemistry, University of Aveiro, 3810-193 Aveiro, Portugal; \\ karynalysenko@ua.pt \\ 2 Ashland Specialty Ingredients, Paul-Thomas Strasse, 56, D-40599 Düsseldorf, Germany; \\ FElSaleh@ashland.com \\ 3 Department of Chemistry, CICECO-Aveiro Institute of Materials, University of Aveiro, \\ 3810-193 Aveiro, Portugal; filipe.paz@ua.pt \\ * Correspondence: sbraga@ua.pt; Tel.: +351-234-370-200 \\ + Presented at the 1st International Electronic Conference on Pharmaceutics, 1-15 December 2020; \\ Available online: https://iecp2020.sciforum.net/.
}

Citation: Braga, S.S.; Lysenko, K.; El-Saleh, F.; Almeida Paz, F.A. Cyclodextrin-Efavirenz Complexes Investigated by Solid State and Solubility Studies. 2021, 78, 15. https://doi. org/10.3390/IECP2020-08690

Published: 1 December 2020

Publisher's Note: MDPI stays neutral with regard to jurisdictional claims in published maps and institutional affiliations.

Copyright: (C) 2020 by the authors. Licensee MDPI, Basel, Switzerland. This article is an open access article distributed under the terms and conditions of the Creative Commons Attribution (CC BY) license (http://creativecommons.org/licenses/by/4.0/).

\begin{abstract}
This short paper investigates the solubilizing ability of various cyclodextrins with efavirenz as well as the formation of solid inclusion complexes of efavirenz with $\beta$-CD and $\gamma-C D$. (1) Background: Efavirenz is a non-nucleoside reverse transcriptase inhibitor used as first-line treatment for adult and pediatric human immunodeficiency virus type 1 infection (HIV-1). Belonging to class II of Biopharmaceutical Classification System (BCS), efavirenz is poorly water-soluble. Inclusion into cyclodextrins is a possible strategy for increasing its solubility. (2) Methods: Solubility modulation was investigated by the phase solubility method; inclusion of efavirenz with $\beta$ - and $\gamma$ cyclodextrins was attempted by co-dissolution with co-precipitation; the precipitates were studied by DSC, FT-IR, powder X-ray diffraction and optical microscopy. (3) Results: Solid state analysis of the precipitates shows evidence of separate recrystallization of $\beta$-cyclodextrin and efavirenz, whereas in the case of $\gamma$-cyclodextrin, a single new phase was observed. (4) Conclusion: Results show that the cavity of $\beta$-cyclodextrin is too narrow to accommodate efavirenz and only $\gamma$-cyclodextrin, the largest of native cyclodextrins, is able to form a true inclusion complex with this bulky guest.
\end{abstract}

Keywords: HIV; $\beta$-cyclodextrin; $\gamma$-cyclodextrin; inclusion complexes; solid-state analysis

\section{Introduction}

Efavirenz (EFV), a non-nucleoside reverse transcriptase inhibitor, is a first-line treatment for adult and pediatric human immunodeficiency virus type 1 infection (HIV-1) both in adults and in children. EFV belongs to class II of Biopharmaceutical Classification System (BCS); that is, it is poorly water-soluble and highly permeable [1]. Several approaches to improve efavirenz dissolution are reported, namely the manipulation of polymorphs [1], the use of superdisintegrants [2], solid dispersions [3,4], nano-sized polymeric micelles [5] and complexation with two chemically modified $\beta$-cyclodextrins, RAMEB and $\mathrm{HP} \beta \mathrm{CD}[6]$.

Cyclodextrins (CDs) are naturally occurring cyclic oligosaccharides, formed by degradation of starch by some microorganisms. Cyclodextrins may also be obtained by enzymatic action on starch. The primarily obtained products of both bacterial and enzymatic processes are named native cyclodextrins and they comprise rings with six (á-CD), seven $(\beta-C D)$ and eight $(\gamma-C D)$ á-D-glucose units. Native cyclodextrins are often used as pharmaceutical excipients, having the GRAS status (Generally Regarded as Safe) from the FDA and approval for use also from the EMA. They can be found in a variety of medicines, 
where they typically play the role of solubilizers. Nevertheless, cyclodextrins may also afford additional advantages, such as protecting drugs against hydrolytical instability or photosensitivity and masking the bitter taste of drugs, including that of herein studied EFV.

The lower price of native cyclodextrins makes them attractive hosts for the inclusion of active pharmaceutical compounds such as efavirenz. Moreover, the results of the present report confirm the better solubilizing performance of $\gamma$-CD over the hydroxypropylated derivatives, $(\mathrm{HP} \gamma \mathrm{CD}$ and even $\mathrm{HP} \beta \mathrm{CD})$. We have thus chosen $\gamma$-CD as host for efavirenz regarding the formation of a solid inclusion complex; $\beta-C D$, for its affordability and similarity in cavity diameter to the two previously reported EFV hosts (RAMEB and $\mathrm{HP} \beta \mathrm{CD}[6]$ ), was also tested regarding EFV complexation in the solid state (Figure 1).
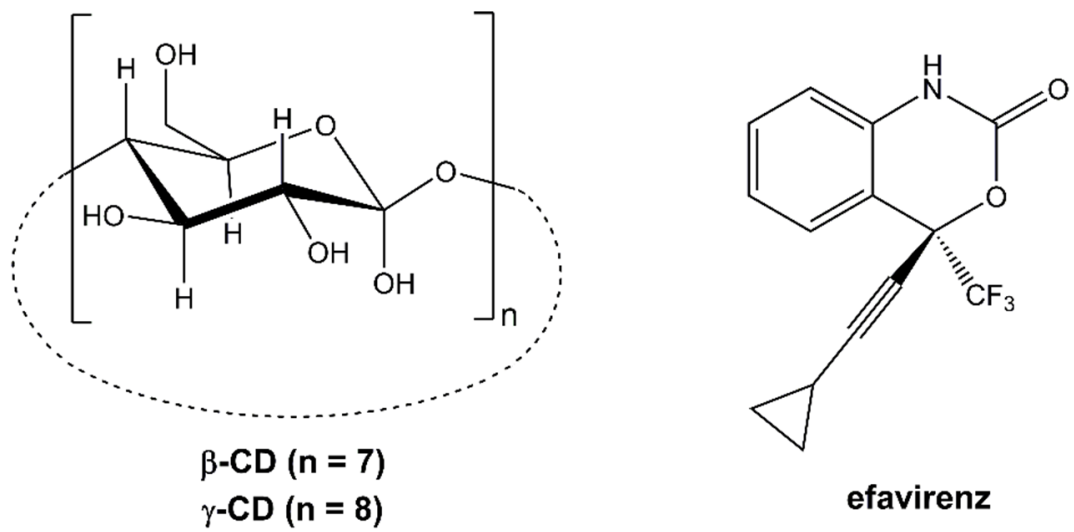

Figure 1. Schematic representation of the $\beta-C D, \gamma-C D$ and efavirenz.

\section{Experiments}

\subsection{Materials}

Pharmaceutical grade $\beta-\mathrm{CD}, \gamma-\mathrm{CD}, \mathrm{HP} \beta \mathrm{CD}$ and $\mathrm{HP} \gamma \mathrm{CD}$ (Cavamax W7 and W8 Pharma, Cavasol W7 HP and Cavasol W8 HP, manufactured by Wacker Chemie) are available at Ashland Specialty Ingredients. Efavirenz was purchased from Smillax. Solvents were of analytical grade unless otherwise specified.

\subsection{Equipment}

UV-Vis data for the solubility isotherms were collected with a Analytik Jena Specord 200 Plus spectrophotometer.

Fourier transform infrared spectra (FT-IR) (range 4000-300 $\mathrm{cm}^{-1}$ ) were collected as $\mathrm{KBr}$ pellets in a Bruker Tensor 27 spectrometer by averaging 128 scans at a $2 \mathrm{~cm}^{-1}$ resolution.

Conventional powder X-ray diffraction (PXRD) data were collected at ambient temperature in a D8 Advance Bruker AXS $\theta-2 \theta$ diffractometer with a copper radiation source (Cu-Ka1, $\lambda=1.5406 \AA$ ) and a secondary monochromator, operated with a flat plate holder in a Bragg-Brentano configuration $(40 \mathrm{kV}$ and $30 \mathrm{~mA})$. Intensity data were collected by the step counting method (step $0.02^{\circ}$ ) in the ca. $3 \leq 2 \theta \leq 40^{\circ}$ range.

DSC samples (5-10 mg), previously desiccated for $72 \mathrm{~h}$ over activated silica gel, were measured in the $20-300{ }^{\circ} \mathrm{C}$ range with $5{ }^{\circ} \mathrm{C} \cdot \mathrm{min}^{-1}$ heating and a $50.0 \mathrm{~mL} \cdot \mathrm{min}^{-1} \mathrm{~N}_{2}$ flow, in a TA instruments DSC Q2000.

\subsection{Solubility Isotherms}

Excess amounts of EFV were added to $20 \mathrm{~mL}$ of unbuffered aqueous solutions of increasing concentrations of tested cyclodextrins. Solutions were stirred for $48 \mathrm{~h}$ at room temperature. Aliquots were filtered $(0.22 \mu \mathrm{m})$, diluted with a water:isopropanol 1:1 mixture and their absorbance was measured at $293 \mathrm{~nm}$. 


\subsection{Inclusion Procedures}

\subsubsection{Inclusion of Efavirenz (EFV) into $\beta-\mathrm{CD}$}

To a solution of $\beta$-CD $(65 \mathrm{mg}, 0.05 \mathrm{mmol})$ in ultrapure water $(2 \mathrm{~mL})$ at $40{ }^{\circ} \mathrm{C}$ was added, dropwise, a solution of EFV $(15.8 \mathrm{mg}, 0.05 \mathrm{mmol})$ in ethanol $(1.5 \mathrm{~mL})$. The mixed solution was stirred for $3 \mathrm{~min}$ and then placed on ice and kept at $3{ }^{\circ} \mathrm{C}$ for $48 \mathrm{~h}$ to induce precipitation of the complex. After decanting, the product was vacuum-dried.

FT-IR v(tilde) $\left(\mathrm{cm}^{-1}\right)=3369 \mathrm{~s}, 2925 \mathrm{~m}, 2251 \mathrm{~m}, 1751 \mathrm{~m}, 1745 \mathrm{sh}, 1603 \mathrm{~m}, 1499 \mathrm{~m}, 1453$ m, 1414 m, 1385 m, 1368 m, 1332 m, 1260 m, 1248 m, 1200 m, 1187 m, 1158 s, 1097 m, 1079 s, $1054 \mathrm{sh}, 1028 \mathrm{vs}, 1005 \mathrm{~s}, 978 \mathrm{~m}, 947 \mathrm{~m}, 928 \mathrm{~m}, 883 \mathrm{w}, 863 \mathrm{w}, 835 \mathrm{w}, 823 \mathrm{w}, 755 \mathrm{~m}, 742 \mathrm{w}$, $706 \mathrm{~m}, 690 \mathrm{~m}, 656 \mathrm{~m}, 607 \mathrm{~m}, 577 \mathrm{~m}, 568 \mathrm{sh}, 556 \mathrm{w}, 530 \mathrm{~m}, 484 \mathrm{w}$.

\subsubsection{Inclusion of Efavirenz (EFV) into $\gamma$-CD}

To a solution of $\gamma-\mathrm{CD}(142 \mathrm{mg}, 0.10 \mathrm{mmol})$ in ultrapure water $(0.7 \mathrm{~mL})$ at $40{ }^{\circ} \mathrm{C}$ was added, dropwise, a solution of EFV $(31.6 \mathrm{mg}, 0.10 \mathrm{mmol})$ in isopropanol with $20 \%$ ethanol $(0.45 \mathrm{~mL})$. The mixed solution was stirred for $3 \mathrm{~min}$ and then placed on ice and kept at 3 ${ }^{\circ} \mathrm{C}$ for $4 \mathrm{~h}$. The precipitate was centrifuged and vacuum-dried.

FT-IR v(tilde) $\left(\mathrm{cm}^{-1}\right)=3390 \mathrm{~s}, 1932 \mathrm{~m}, 2893 \mathrm{sh}, 2252 \mathrm{w}, 1753 \mathrm{~m}, 1637 \mathrm{~m}, 1499 \mathrm{~m}, 1458$ m, 1414 m, 1383 m, 1372 m, 1335 m, 1302 m, 1249 m, 1198 m, 1187 m, 1159 s, 1099 sh, 1079 s, 1052 sh, 1025 vs, 1000 s, 942 m, 931 sh, 861 w, 838 vw, 760m, 742 w, 706 m, 691 w, 674 vw, $671 \mathrm{vw}, 655 \mathrm{w}, 608 \mathrm{w}, 580 \mathrm{~m}, 566 \mathrm{sh}, 528 \mathrm{w}, 480 \mathrm{w}$.

\section{Results and Discussion}

\subsection{Phase Solubility Studies}

Three different cyclodextrins, $\gamma-\mathrm{CD}, \mathrm{HP} \beta \mathrm{CD}$ and $\mathrm{HP} \gamma \mathrm{CD}$, were tested regarding their ability to increase the aqueous solubility of EFV. Results of the phase solubility study can be seen in Figure 2.

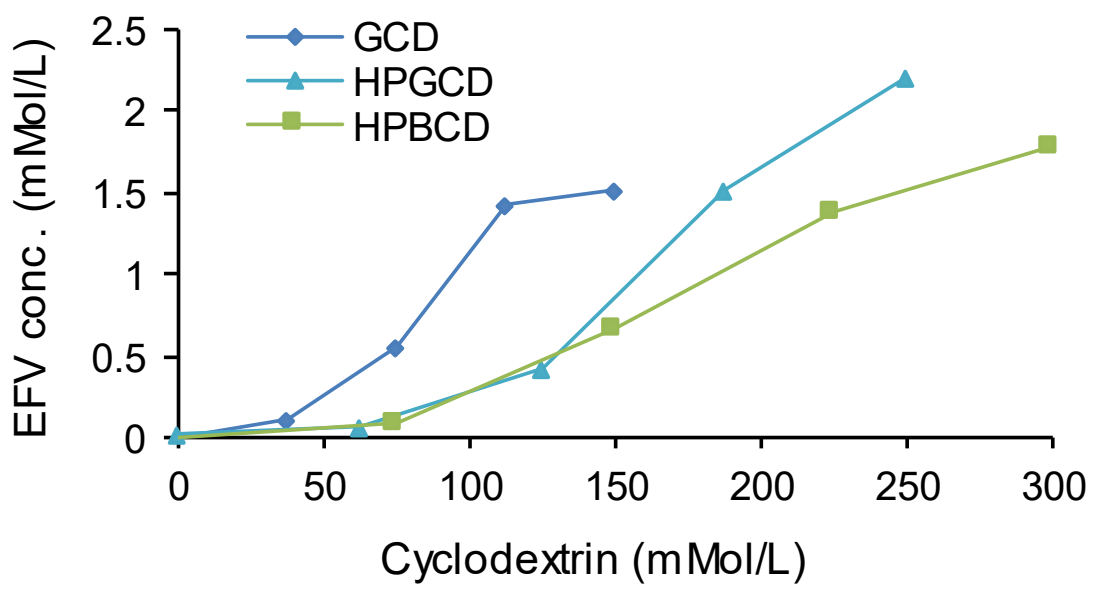

Figure 2. Phase solubility isotherms of EFV in the presence of $\gamma$-CD (blue diamonds, GCD), $\mathrm{HP} \beta \mathrm{CD}$ (turquoise triangles, HPBCD), and $\mathrm{HP} \gamma \mathrm{CD}$ (green squares, HPGCD).

Interestingly, the native cyclodextrin, $\gamma-\mathrm{CD}$, was the most effective in solubilizing $\mathrm{EFV}$, followed by $\mathrm{HP} \gamma \mathrm{CD}$ and then HP $\beta \mathrm{CD}$. The non-linear form of the graphs suggests that some higher ordered aggregates might have been formed. This phenomenon is well known for $\gamma$-CD. Note also that the results herein presented confirm the findings of Sathigari et al. [6] regarding HP $\beta C D$ and suggest that $\gamma$-CD should be the most suitable cyclodextrin to form a complex with EFV, probably due to its larger cavity that can accommodate a larger portion of the EFV molecule. 


\subsection{Preparation and Visual Inspection of Cyclodextrin-Efavirenz Co-Precipitates}

EFV was co-dissolved with each cyclodextrin $(\beta-C D$ and $\gamma-C D)$ for 3 min and then the mixture was co-precipitated under cooling. An early investigation into the products of each precipitation was conducted with optical microscopy. Images are depicted in Figure 3.
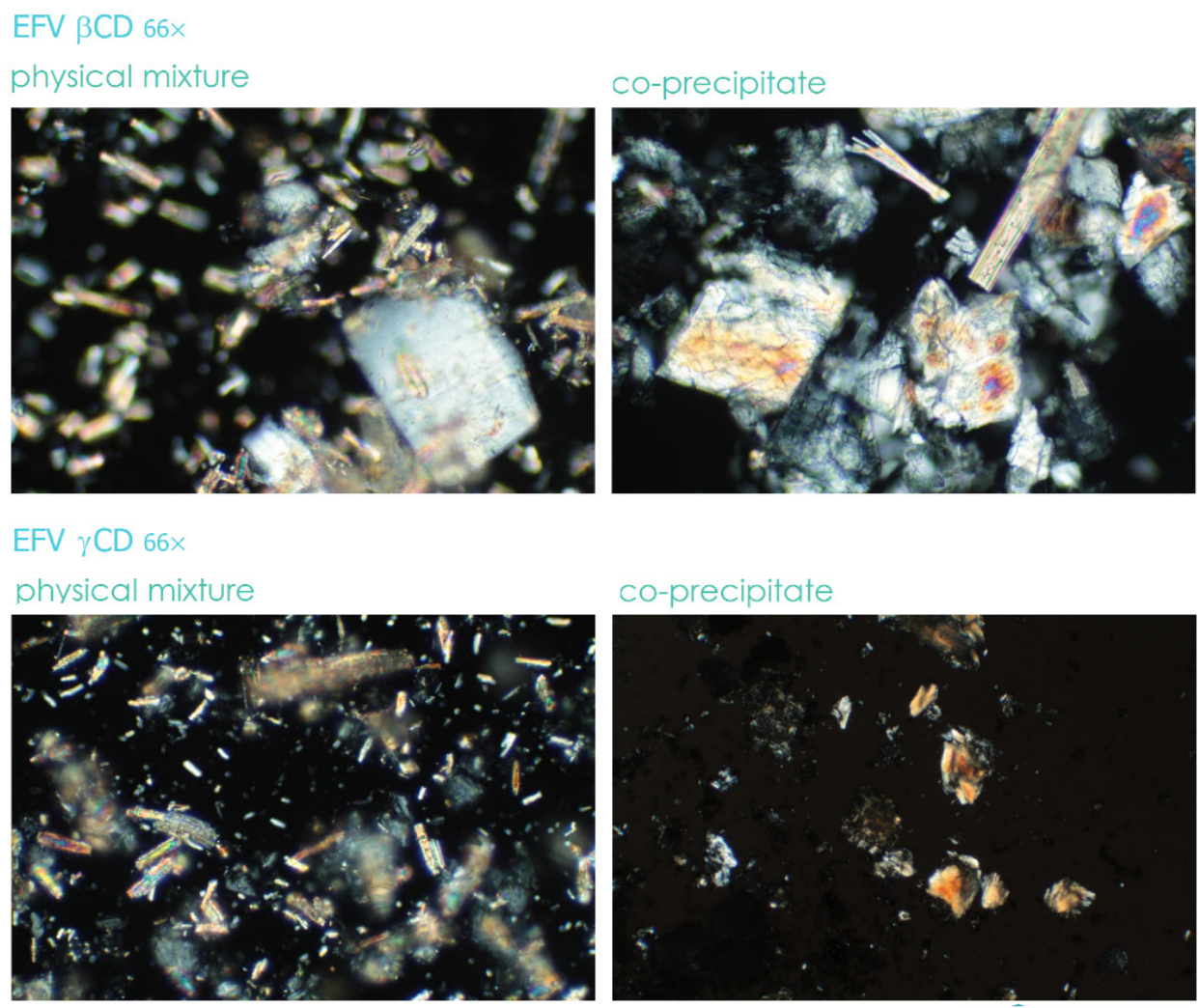

Figure 3. Optical microscopy photographs, at 66 times magnification, of the precipitates obtained from co-dissolving and co-precipitating efavirenz with $\beta$-CD (top right) and $\gamma-C D$ (bottom right); on the left side of the figure are shown the corresponding physical mixtures, for comparison of particle size and morphology.

A visual comparison of the co-precipitate obtained from the batch of $\beta$-CD with efavirenz permits noting the presence of two types of particles, small needles and larger parallelepipeds, which bear striking similarities with the materials observed in the image of the physical mixture and thus allow concluding that the sample contains the two recrystallized starting materials, efavirenz and $\beta$-CD. In turn, the image of the co-precipitate obtained from the batch of $\gamma$-CD with efavirenz shows the lack of the needles attributed to this guest and the formation of a new and seemingly glassy material with irregular shapes. This observation is a first indication on the inclusion of efavirenz into $\gamma-C D$, which can be confirmed by the analyses described in the following subsections.

\subsection{FT-IR of the Co-Precipitates}

The FT-IR absorption bands of EFV appear mostly unshifted in the products $\beta$ CD.EFV and $\gamma$-CD.EFV, with exception of the $C=O$ stretch that is blue shifted. In $\beta$ $\mathrm{CD} \cdot \mathrm{EFV}$, it appears at 1751 with a small shoulder at $1745 \mathrm{~cm}^{-1}$ (note that in pure EFV vco $=1742 \mathrm{~cm}^{-1}$ ), and in $\gamma$-CD.EFV, it peaks at $1743 \mathrm{~cm}^{-1}$. The increased energy of this oscillator denotes the disruption of intermolecular hydrogen bonds occurring in pure EFV [1], either due to inclusion into the $\mathrm{CD}$ cavity or by formation of a new bond with an $\mathrm{OH}$ group at the 
rim of the $\mathrm{CD}$ (which may not necessarily be associated with the formation of an inclusion compound).

\subsection{Powder X-ray Diffraction of the Co-Precipitates}

The PXRD pattern of EFV is characteristic of its polymorph II, the most stable form at ambient temperature [1]. The diffractogram of $\beta$-CD-EFV is dominated by reflections associated with the host and also a few from EFV. The absence of a new crystalline phase in tandem with the presence of the phases associated with the starting materials clearly indicates that inclusion did not take place. The PXRD data for this host-guest-system is also in agreement with the observations made from the analysis of sample morphology under the optical microscope.

In turn, the PXRD trace of $\gamma$-CD-EFV shows the presence of more amorphous material and a few broad reflections, peaking at 6.2, 7.5, 10.6, 11.9, and 16.2 degrees of $2 \theta$, that do not correspond to either pure EFV or $\gamma$-CD (Figure 4).

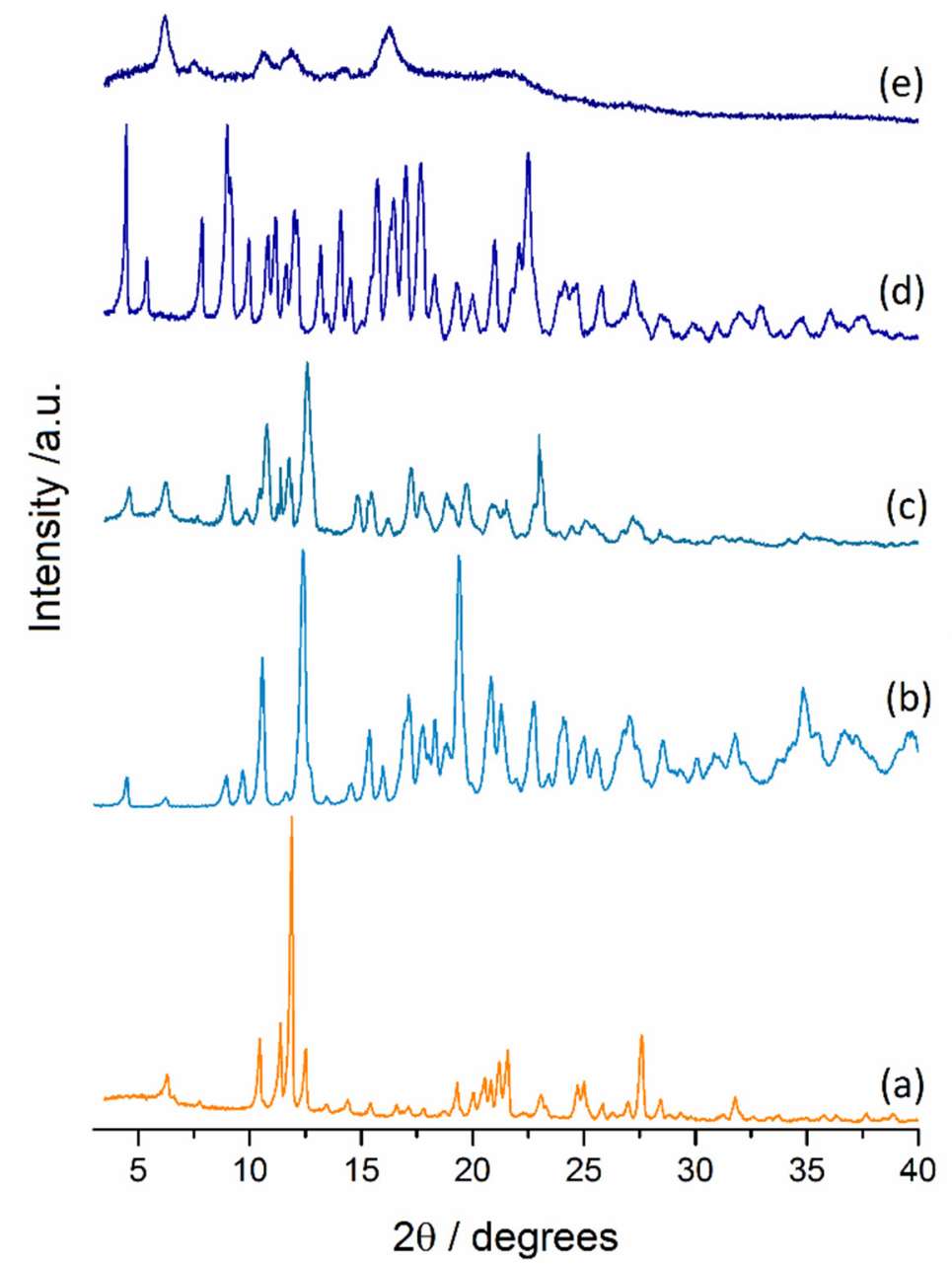

Figure 4. Powder XRD patterns of (a) efavirenz; (b) $\beta$-CD hydrate; (c) $\beta$-CD-EFV co-precipitate; (d) $\gamma$-CD heptahydrate; (e) $\gamma$-CD-EFV co-precipitate.

\subsection{DSC Analysis}

DSC traces of the co-precipitates, their components, $\beta-C D, \gamma-C D$ and EFV, and the corresponding 1:1 physical mixtures are presented in Figure 5. The trace of pure EFV shows a melting peak at $138^{\circ} \mathrm{C}$, which is also present in the DSC trace of the sample coprecipitated from the co-dissolved $\beta$-CD and EFV. Note also that this melting peak presents a shape and intensity identical to the peak observed in the 1:1 PM of $\beta C D$ and EFV, thus confirming that the co-precipitate is in fact no different from a physical mixture. 
In the co-precipitate of $\gamma$-CD.EFV, the melting peak is centered at $137^{\circ} \mathrm{C}$ and it is broader due to amorphization; also noteworthy is the lower number of hydration water molecules in this sample.

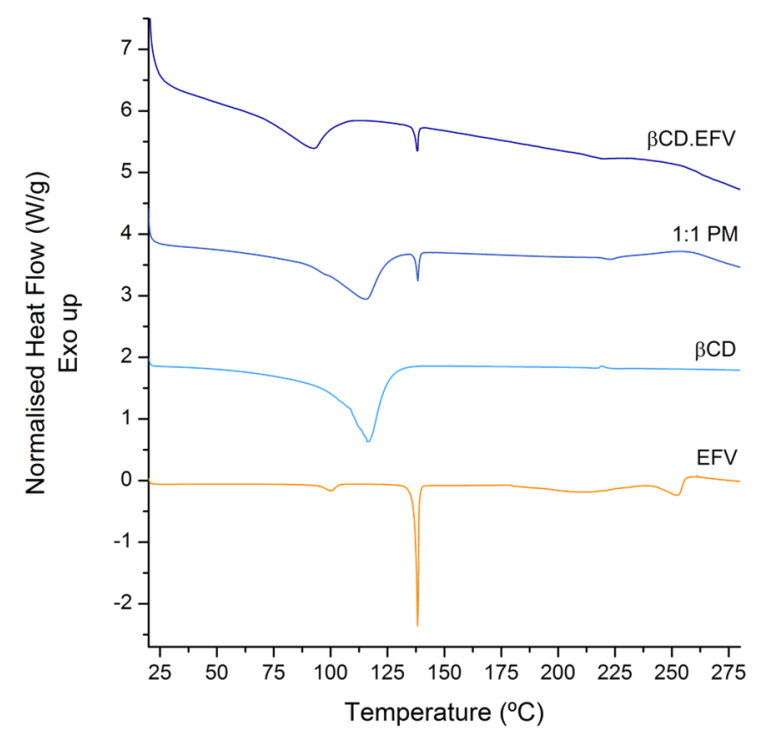

(a)

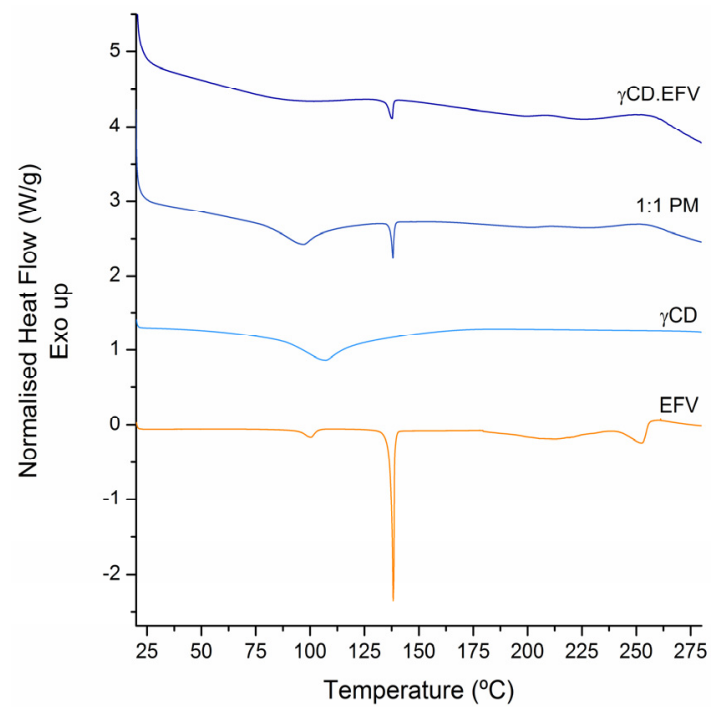

(b)

Figure 5. DSC of the EFV co-precipitate products with (a) $\beta$-CD and (b) $\gamma$-CD.

\section{Conclusions}

The interaction of EFV with native $\gamma-\mathrm{CD}$ and two hydroxypropylated derivatives, $\mathrm{HP} \beta \mathrm{CD}, \mathrm{HP} \gamma \mathrm{CD}$ was observed in aqueous solution through the enhancement of its solubility values. The strongest solubilization of enhancement was observed with $\gamma-C D$ at concentrations up to $0.1 \mathrm{M}$, with the formation of large molecular aggregates leading to a plateau in the value of dissolved EFV after this point.

The formation of CD inclusion compounds of EFV in the solid state was also investigated, using the native $\beta$-CD and $\gamma$-CD hosts. The results of microscopy, powder XRD and DSC indicate that EFV interaction with $\beta$-CD does not lead to the formation of a stable solid compound, with EFV and $\beta$-CD recrystallizing as two separate phases. In turn, EFV interaction with $\beta$-CD leads to the formation of a true inclusion compound, albeit the coprecipitation method herein employed did not allow for a highly crystalline material to be isolated. Future work will follow up on these findings and optimize the inclusion method for the preparation of $\gamma$-CD.EFV in higher amounts. Crystallization procedures will also be explored, aiming at the isolation of $\gamma$-CD.EFV in the crystalline form.

Data Availability Statement: Solubility/dissolution, microscopy images and DSC data are proprietary of Ashland Specialty Ingredients.; solid-state characterisation data are available upon request from the diffraction services of the University of Aveiro.

Acknowledgments: We acknowledge University of Aveiro and Fundação para a Ciência e Tecnologia/MCTES for the financial support to the associated laboratory LAQV-REQUIMTE (project reference UIDB/50006/2020) and to CICECO-Aveiro Institute of Materials (UIDB/50011/2020 and UIDP/50011/2020), through national funds and, where applicable, co-financed by the FEDER, within the PT2020 Partnership Agreement, and to the Portuguese NMR Network.

Conflicts of Interest: The authors declare no conflict of interest. 


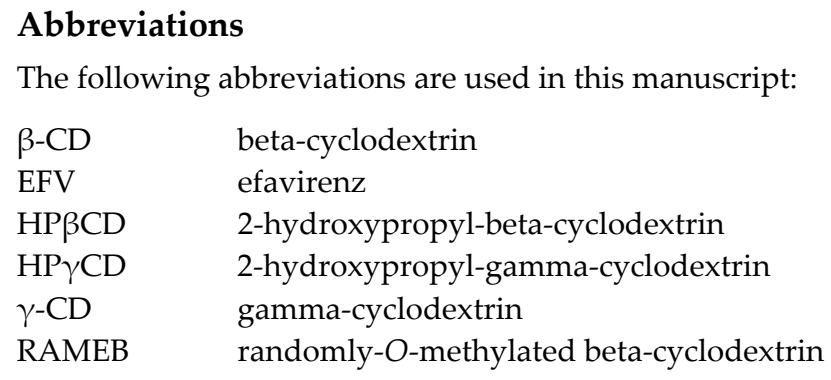

\section{References}

1. Fandaruff, C.; Rauber, G.S.; Araya-Sibaja, A.M.; Pereira, R.N.; de Campos, C.E.M.; Rocha, H.V.A.; Monti, G.A.; Malaspina, T.; Silva, M.A.S.; Cuffini, S.L. Polymorphism of anti-HIV drug efavirenz: Investigations on thermodynamic and dissolution properties. Cryst. Growth Des. 2014, 14, 4968-4974, doi:10.1021/cg500509c.

2. Rajesh, Y.V.; Balasubramaniam, J.; Bindu, K.; Sridevi, R.; Swetha, M.; Rao, V.U. Impact of superdisintegrants on efavirenz release from tablet formulations. Acta Pharm. 2010, 60, 185-195, doi:10.2478/v10007-010-0019-6.

3. Yang, J.; Grey, K.; Doney, J. An improved kinetics approach to describe the physical stability of amorphous solid dispersions. Int. J. Pharm. 2010, 384, 24-31, doi:10.1016/j.ijpharm.2009.09.035.

4. Madhavi, B.B.; Kusum, B.; Charanya, C.K.; Madhu, M.N.; Harsa, V.S.; Banji, D. Dissolution enhancement of efavirenz by solid dispersion and PEGylation techniques. Int. J. Pharm. Investig. 2011, 1, 29-34, doi:10.14227/DT210214P31.

5. Chiappetta, D.A.; Hocht, C.; Taira, C.; Sosnik, A. Efavirenz-loaded polymeric micelles for pediatric anti-HIV pharmacotherapy with significantly higher oral bioavailability. Nanomedicine 2010, 5, 11-23, doi:10.2217/nnm.09.90.

6. Sathigari, S.; Chadha, G.; Lee, Y.L.; Wright, N.; Parsons, D.L.; Rangari, V.K.; Fasina, R.; Babu, J. Physicochemical characterization of efavirenz cyclodextrin inclusion complexes. AAPS PharmSciTech 2009, 10, 81-87, doi:10.1208/s12249-008-9180-3. 